\title{
Developments from Ernst Specker's Work in Set Theory
}

\author{
Andreas Blass ${ }^{1}$ \\ Andreas Blass graduated from the University of Detroit, where he was a Putnam Fel- \\ low, in 1966 with a B.S. in physics. He received his Ph.D. in 1970 from Harvard \\ University with a thesis on orderings of ultrafilters. He is currently a professor at the \\ University of Michigan. His research is primarily in mathematical logic, particularly \\ set theory, but it extends into other areas, including finite combinatorics, category the- \\ ory, and theoretical computer science.
}

I first met Ernst Specker at the 2010 Zürich conference in honor of his 90th birthday, so I am not in a position to contribute reminiscences. Like all set theorists, however, I met Specker's mathematics early and often in my mathematical career. At the Zürich meeting I reported briefly on some of his contributions to set theory and on some recent developments that build on those contributions. I thank Norbert Hungerbühler and János Makowsky for giving me the opportunity to contribute this note, based on my Zürich talk.

The time constraints of the talk and the space constraints of this note require me to limit myself to just three of Specker's set theory papers. Each of the three sections that follow concerns one of the papers and the subsequent developments flowing from it.

\section{Sur un problème de Sikorski}

Unpublished work of Specker closely related to this paper [18] showed the existence of Specker lines. These are linearly ordered sets of cardinality $\aleph_{1}$ such that no uncountable subset is well-ordered, anti-well-ordered, or embeddable into the real line $\mathbb{R}$ (with the usual order).

1. Partially supported by NSF grant DMS-0653696

Ernst Specker hat richtungsweisende Beiträge in verschiedensten Gebieten der Mathematik geleistet: Topologie, Algebra, Mathematische Logik, Mengenlehre, Kombinatorik und Algorithmik. Es ist nicht möglich einen kurzen Überblick über sein Werk zu geben. Im vorliegenden Beitrag zeigt Andreas Blass jedoch für drei ausgewählte Arbeiten von Ernst Specker zur Mengenlehre auf, welche weiteren Entwicklungen die darin enthaltenen Resultate initiiert haben. 
The existence of Specker lines was the key to the proof by Galvin and Shelah [8] of the negative partition relation

$$
\aleph_{1} \nrightarrow\left[\aleph_{1}\right]_{4}^{2}
$$

That is, one can partition the set of 2-element subsets of a set $A$ of size $\aleph_{1}$ into four pieces in such a way that every uncountable subset of $A$ contains pairs from all four pieces. Indeed, such a partition can be obtained by choosing three linear orderings of $A$ : a wellordering $\leq_{1}$, an ordering $\leq_{2}$ embeddable into $\mathbb{R}$, and an ordering $\leq_{3}$ as a Specker line. The two-element subsets $\{a, b\}$ of $A$ are then classified according to which of $\leq_{1}, \leq_{2}$, and $\leq_{3}$ agree on $\{a, b\}$. It is easy to see that any uncountable subset must contain pairs from at least three of the four classes; with more work, Galvin and Shelah improved this from three to all four.

Without a Specker line, this approach would yield only the weaker, classical theorem of Sierpiński [16] that $\aleph_{1} \nrightarrow\left[\aleph_{1}\right]_{2}^{2}$. Later, a different approach enabled Todorčević [21] to prove the strongest possible result of this sort, $\aleph_{1} \nrightarrow\left[\aleph_{1}\right]_{\aleph_{1}}^{2}$.

Saharon Shelah [15] solved a long-standing problem by proving the existence of a Countryman line, i.e., a linear order $\langle L, \leq\rangle$ of cardinality $\aleph_{1}$ such that $L \times L$, with the componentwise partial order, is the union of countably many chains. It is not hard to see that every Countryman line is a Specker line. It is even easier to see that there cannot be an isomorphism between an uncountable subset of a Countryman line and a subset of the same line with the reverse order. (The graph of an isomorphism, as a subset of $L \times L$, would meet each chain of $L \times L$ at most once.) These facts make Countryman lines an important ingredient of the basis problem for uncountable linear orders: How large must a family $\mathcal{B}$ of linear orders of size $\aleph_{1}$ be so that every linear order of size $\aleph_{1}$ contains an isomorphic copy of one from $\mathcal{B}$ ?

The generalized continuum hypothesis $(\mathrm{GCH})$ implies that the smallest possibility for $|\mathcal{B}|$ is $2^{\aleph_{1}}$. A set of size $\aleph_{1}$ has, under $\mathrm{GCH}, 2^{\aleph_{1}}$ linear orderings no two of which have uncountable subsets that are isomorphic.

In contrast to this, Justin Moore [11] proved that it is consistent (relative to large cardinals) and it follows from the proper forcing axiom that there is such a basis $\mathcal{B}$ with just five members:

$-\omega_{1}$ (the shortest well-ordering of cardinality $\aleph_{1}$ ),

$-\omega_{1}^{*}\left(\omega_{1}\right.$ with the ordering reversed),

- an $\aleph_{1}$-sized subset of $\mathbb{R}$,

- a Countryman line $C$, and

$-C^{*}$, the same Countryman line with the reverse order.

This result is optimal, since no two of these five orderings have uncountable subsets that are isomorphic. 


\section{Additive Gruppen von Folgen ganzer Zahlen}

This paper [19] contains some (still) surprising ${ }^{2}$ properties of the additive group

$$
\Pi=\mathbb{Z}^{\mathbb{N}}
$$

of all sequences of integers and its monotone subgroups. "Monotone" means that, if a sequence $\left\langle x_{n}\right\rangle$ is in the subgroup $M$ and if $\left\langle y_{n}\right\rangle \in \Pi$ satisfies $\left|y_{n}\right| \leq \max \left\{1,\left|x_{k}\right|: k \leq n\right\}$ for all $n$, then $\left\langle y_{n}\right\rangle \in M$. The smallest monotone subgroup is the group $\mathcal{B}$ of bounded sequences; the largest is $\Pi$ itself.

Specker proved that, for any monotone $M$ other than $\mathcal{B}$, every homomorphism $h: M \rightarrow \mathbb{Z}$ factors through a finite subproduct. That is, $h$ has the form

$$
h\left(\left\langle x_{n}\right\rangle\right)=\sum_{n=0}^{r} a_{n} x_{n}
$$

for some finite $r$ and some integers $a_{0}, \ldots, a_{r}$.

It follows that $M$ is not free. Indeed, since $M$ has the cardinality $\mathfrak{c}$ of the continuum, any free basis for it would have the same cardinality, and then any of the $2^{\mathfrak{c}}$ functions from the basis to $\mathbb{Z}$ would extend (uniquely) to a homomorphism. But Specker's result shows that $M$ admits only countably many homomorphisms to $\mathbb{Z}$, two exponentials lower than the $2^{\mathfrak{c}}$ that it would have if it were free.

Specker also showed that the continuum hypothesis $(\mathrm{CH})$ implies that $\mathcal{B}$ is free. Later, Nöbeling [14] removed the need for $\mathrm{CH}$. Nevertheless, the proof makes essential use of the axiom of choice, and in fact all "well-behaved" homomorphisms $\mathcal{B} \rightarrow \mathbb{Z}$ factor through a finite subproduct [2]. Here the "well-behaved" homomorphisms include all those that are Borel with respect to the topology that $\mathcal{B}$ inherits as a subspace of the product $\Pi$ of discrete spaces $\mathbb{Z}$. And it is consistent, in the absence of the axiom of choice, that all homomorphisms are well-behaved.

All monotone subgroups of $\Pi$ have cardinality $c$. Could smaller subgroups (still of infinite rank) have the same property that all homomorphisms to $\mathbb{Z}$ factor through a finite subproduct? Countable subgroups of $\Pi$ are free, so they cannot have this factorization property, but, in the absence of $\mathrm{CH}$, one can ask about subgroups of cardinality strictly between $\aleph_{0}$ and $\mathfrak{c}$. Katsuya Eda [5] showed that the answer is independent of ZFC plus not-CH. I subsequently showed [1] that, if $\kappa$ is the cardinality of a subgroup of $\Pi$ with this factorization property, then there are $\kappa$ sets in $\mathbb{R}$ of Lebesgue measure zero whose union does not have Lebesgue measure zero. (The underlying combinatorics, which connects the factorization property to Lebesgue measure, has appeared again in other contexts.)

Generalizing a notion introduced by Łoś, call an abelian group $G$ slender for a monotone subgroup $M$ of $\Pi$ if every homomorphism $M \rightarrow G$ factors through a finite subproduct. So Specker's theorem says that $\mathbb{Z}$ is slender for all $M$ except $\mathcal{B}$.

2. A few years ago, I gave a colloquium talk in my department, and mentioned, in the abstract circulated beforehand, that $\Pi$ is not free. Two of my colleagues independently came to my office to ask whether I really meant that, since it seemed clear to them that $\Pi$ must be free. 
There are $2^{\mathfrak{c}}$ monotone subgroups of $\Pi$. How many notions of slenderness do they produce? Göbel and Wald [9] showed that there are at leat four. Assuming Martin's Axiom, they got the maximum possible number, $2^{\mathfrak{c}}$. But it is consistent [3] that there are only four.

There are combinatorial methods, called $\Gamma$-invariants, for measuring how far an abelian group is from being free; see for example [6]. By this measure, monotone subgroups of $\Pi$ other than $\mathcal{B}$ are as far from free as possible.

\section{Zur Axiomatik der Mengenlehre (Fundierungs- und Auswahlaxiom)}

Part II of this paper [20] contains a reformulation and abstraction of the Fraenkel-Mostowski method of permutation models for the negation of the axiom of choice. Fraenkel [7] and Mostowski [12] worked with a version of set theory that allows atoms (also called Urelemente), which are not sets but can be members of sets. A universe $V$ of sets built over a family $A$ of atoms admits automorphisms induced by arbitrary permutations of $A$. Permutation models $M$ are composed of the atoms together with some, not all, of the sets that can be formed from them; sets are allowed into $M$ if they (and their elements, elements of elements, etc.) are sufficiently symmetric, i.e., invariant under enough automorphisms of $V$. Specker introduced two modifications of the Fraenkel-Mostowski method. The lesser of the two was to replace the atoms, which are not sets, with sets of a special sort, namely ones that satisfy $a=\{a\}$. The effect is to reinstate the axiom of extensionality, "things with the same members are equal," which had been violated by the atoms. The price for this reinstatement is that one loses the axiom of foundation, which prohibits, among other things, sets having themselves as members. The net effect is a gain, since extensionality plays a far larger role in mathematics than foundation.

The more important of Specker's modifications concerns the notion of invariance under "enough" automorphisms. In the work of Fraenkel and Mostowski, "enough automorphisms" meant "all automorphisms induced by permutations that fix a small family of atoms and preserve some additional structure on the atoms"; the notion of "small family" and the relevant additional structure were chosen, in each particular construction, to accomplish the desired technical results, for example verifying some weak version of the axiom of choice in $M$. Specker saw that both smallness and extra structure can be more naturally expressed in terms of the group of permutations and a filter of subgroups. $\mathrm{He}$ made this group-theoretic viewpoint the center of his development of the theory, thereby achieving the proper, natural level of abstraction for this work.

Later, when Cohen [4] proved the independence of the axiom of choice from full ZermeloFraenkel set theory (including the axioms of extensionality and foundation), the grouptheoretic viewpoint was still used, but, instead of permuting atoms (or self-member sets), the group acted by automorphisms on the forcing conditions used in constructing and describing Cohen's models. The group-theoretic viewpoint made evident an analogy between Cohen's constructions and the earlier Fraenkel-Mostowski-Specker permutation models.

It turns out, however, that there is more than an analogy here; Cohen's models can be constructed directly, by forcing over permutation models, with no additional use of symmetry. This approach is presented in [22]. Here is an example: Start with the basic Fraenkel 
model (as in [7] or [10, Chapter 4]) with its set $A$ of "atoms" $a=\{a\}$. Adjoin, in the usual way, an $A$-indexed family of Cohen-generic reals, by forcing with finite partial functions $A \times \omega \rightarrow 2$. The well-founded part of the resulting forcing extension is the basic Cohen model (as in [4] or [10, Chapter 5]).

In Part III of the same paper [20], Specker analyzed various properties of ordinals that become possible when the axiom of choice is not assumed. He showed that some of these situations imply that the first uncountable ordinal $\omega_{1}$ is, in Gödel's constructible universe, an inaccessible cardinal. In particular, he deduced this conclusion from the hypothesis that there is no function assigning, to each countable limit ordinal $\alpha$, an $\omega$-sequence of smaller ordinals converging to $\alpha$. (Every countable limit ordinal $\alpha$ admits such an $\omega$-sequence; the issue is whether there is a function choosing one such $\omega$-sequence for each $\alpha$.) This hypothesis therefore has consistency strength at least that of an inaccessible cardinal, strictly greater than $\mathrm{ZF}$ alone.

It turns out that this hypothesis holds in some of the most important models of ZF without choice, including Solovay's model [17] for "all sets of reals are Lebesgue measurable" (which has exactly the consistency strength of an inaccessible cardinal) and models of Mycielski's axiom of determinacy [13] (which has considerably higher consistency strength).

\section{References}

[1] Andreas Blass, "Cardinal characteristics and the product of countably many infinite cyclic groups," $J$. Algebra 169 (1994) 512-540.

[2] Andreas Blass, "Specker's theorem for Nöbeling's group," Proc. Amer. Math. Soc. 130 (2002) 1581-1587.

[3] Andreas Blass and Claude Laflamme, "Consistency results about filters and the number of inequivalent growth types," J. Symbolic Logic 54 (1989) 50-56.

[4] Paul Cohen, "The independence of the continuum hypothesis," Part I, Proc. Natl. Acad. Sci. USA 50 (1963) 1143-1148; Part II, ibid. 51 (1964) 105-110.

[5] Katsuya Eda, “A note on subgroups of $Z^{N}$," Abelian Group Theory, Proceedings, Honolulu 1982/83, (ed. R. Göbel, L. Lady, A. Mader) Springer-Verlag, Lecture Notes in Mathematics 1006 (1983) 371-374.

[6] Paul Eklof and Alan Mekler, Almost Free Modules: Set-Theoretic Methods, North-Holland Mathematical Library 46 (1990).

[7] Abraham Fraenkel, "Der Begriff “definit” und die Unabhängigkeit des Auswahlaxioms," Sitzungsberichte der Preußischen Akademie der Wissenschaften, Physikalisch-Mathematische Klasse (1922) 253-257.

[8] Fred Galvin and Saharon Shelah, "Some counterexamples in the partition calculus," J. Combin. Theory Ser. A 15 (1973) 167-174.

[9] Rüdiger Göbel and Burkhard Wald, "Martin's axiom implies the existence of certain slender groups," Math. Z. 172 (1980) 107-121.

[10] Thomas Jech, The Axiom of Choice, North-Holland, Studies in Logic and the Foundations of Mathematics 75 (1973).

[11] Justin Tatch Moore, "A five element basis for the uncountable linear orders," Ann. Math., Ser. 2163 (2006) 669-688.

[12] Andrzej Mostowski, "Über die Unabhängigkeit des Wohlordnungssatzes vom Ordnungsprinzip," Fund Math. 32 (1939) 201-252.

[13] Jan Mycielski, "On the axiom of determinateness," Fund. Math. 53 (1964) 205-224.

[14] Georg Nöbeling, "Verallgemeinerung eines Satzes von Herrn E. Specker," Invent. Math. 6 (1968) 41-55.

[15] Saharon Shelah, "Decomposing uncountable squares to countably many chains," J. Combin. Theory Ser. A 21 (1976) 110-114. 
[16] Wacław Sierpiński, "Sur un problème de la théorie des relations," Ann. Sc. Norm. Super. Pisa, Ser. 22 (1933) 285-287

[17] Robert Solovay, "A model of set theory in which every set of reals is Lebesgue measurable," Ann. Math., Ser. 292 (1970) 1-56.

[18] Ernst Specker, "Sur un problème de Sikorski," Colloquium Math. 2 (1949) 9-12.

[19] Ernst Specker, “Additive Gruppen von Folgen ganzer Zahlen,” Portugaliae Math. 9 (1950) 131-140.

[20] Ernst Specker, "Zur Axiomatik der Mengenlehre (Fundierungs- und Auswahlaxiom)," Z. Math. Logik Grundlagen Math. 3 (1957) 173-210.

[21] Stevo Todorčević, "Partitioning pairs of countable ordinals," Acta Math. 159 (1987) 261-294.

[22] Petr Vopěnka and Petr Hájek, The Theory of Semisets, Academia (1972).

\section{Andreas Blass}

Mathematics Department

University of Michigan

Ann Arbor, MI 48109-1043, USA

e-mail: ablass@umich.edu 